\title{
La importancia del temperamento en la producción de ganado de carne bovina
}

\author{
The importance of temperament in beef cattle production
}

\section{A importância do temperamento na produção de gado bovino de corte}

\author{
Leandro M. León-Llanos ${ }^{1 *}$, Hernando Flórez-Díaz ${ }^{2 *}$ \\ 1* MVZ, MSc, Corpoica La Libertad. Kilómetro 21 vía Puerto López. \\ 2* MVZ, MSc, PhD, Corpoica La Libertad. Kilómetro 21 vía Puerto López. \\ Villavicencio - Meta, Colombia \\ Email: hflorez@corpoica.org.co
}

Recibido: febrero 21 de $2015 \quad$ Aceptado: noviembre 3 de 2016

\begin{abstract}
Resumen
En las explotaciones ganaderas, los bovinos son expuestos a diferentes factores estresantes desde el crecimiento hasta el sacrificio, afectando el bienestar y temperamento de los animales. El término "temperamento" se define como la reacción producida en un animal ante la presencia del hombre y se encuentra afectado por diferentes factores como la raza, el género, la edad, el manejo y la genética. Durante la evaluación del temperamento los bovinos excitables (nerviosos) tienden a presentar resultados altos en las evaluaciones de corral (EC), brete (EB) y velocidad de salida del brete (VSB), los cuales están relacionados con concentraciones elevadas de glucocorticoides y catecolaminas. Asimismo, los animales de temperamento nervioso presentan bajas ganancias diarias de peso (crecimiento lento) y bajos resultados de calidad de la canal y de la carne en comparación con animales de temperamento calmado. Por lo que el propósito de este documento fue revisar la relación entre el temperamento y la producción de carne bovina.
\end{abstract}

Palabras clave: Calidad de la canal, Estrés, Ganancia de Peso, Sacrificio, Terneza, Velocidad de Salida.

\begin{abstract}
In livestock farms, cattle are exposed to different stressors from growth to slaughter, affecting the welfare and temperament of animals. The term "temperament" is defined as the reaction produced by an animal in the presence of man and is affected by various factors such as breed, gender, age, management and genetics. During the evaluation of cattle temperament, animals with an excitable temperament (nervous) tend to have high scores on assessments of pen, chute and chute exit velocity, which are associated with high concentrations of glucocorticoids and catecholamines. Also, animals with nervous temperament have low daily gains (slow growth) and lower results of carcass and meat quality compared to animals with calm temperament. The purpose of this paper is to review the relationship between temperament and beef cattle production.
\end{abstract}

Keywords: Carcass Quality, Stress, Weight Gain, Slaughter, Exit Velocity, Tenderness. 


\section{Resumo}

Nas fazendas pecuarias o gado esta exposto a diferentes estressores do crescimento ate a idade de sacrificio, afetando o bem-estar e temperamento dos animais. O termo "temperamento" é definido como a reacção produzida num animal perante a presença de homem e é afectada por factores como a raça, sexo, idade, manejo e genética. Durante a evaluacao de os bovinos excitáveis (nervosos) tendem a apresentar resultados maiores nas avaliações de curral (EC) brete (EB) e velocidade de saída do brete (VSB), as quais estão relacionados com altas concentrações de glicocorticóides e catecolaminas . Além disso, animais de temperamento nervoso têm baixo ganho de peso diário (de crescimento lento), e baixos resultados de qualidade de carcaça e carne em comparação com animais temperamento calmo. Assim, o objetivo deste trabalho foi avaliar a relação entre temperamento e produção de carne bovina.

Palavras-chave: Qualidade da carcaça, stress, ganho de peso, abate, velocidade de saída

\section{Introducción}

En Colombia la industria ganadera se ha visto obstaculizada por los bajos procesos de modernización en los diferentes eslabones de la cadena cárnica, ocasionados por el atrasado sistema de comercio, el castigo comercial por la presencia de cruces con Bos taurus, la elevada edad para el sacrificio y la persistencia de ferias comerciales para la venta de ganado gordo, los cuales son aspectos que deben ser corregidos con el fin de ajustarlos al contexto mundial.

En el territorio nacional el $80 \%$ de ganado bovino corresponde a ganado Cebú comercial (Santana D, 2009), seguidos de la ganadería doble propósito que incluyen cruces Bos taurus x Bos indicus en diferentes proporciones y los sistemas de lechería especializada que se basa en el ganado Holstein principalmente (León-Llanos, 2009).

En la región de la Orinoquia, predominan las razas cebuínas principalmente el Brahman y en menor proporción Guzerat, Gyr, Nelore y los cruces entre estas, dando como resultado un ganado Cebú comercial. Observando en menor proporción cruces de razas cebuínas con ganados europeos o criollos (León-Llanos, 2009).

Una de las ventajas por las que se caracteriza el ganado Cebú, es la facilidad de adaptarse a las condiciones tropicales; sin embargo, se ha observado que son animales de temperamento nervioso en comparación con bovinos Bos taurus (Crouse et al., 1989). Para el propósito de esta revisión, el término "temperamento" se define como la reacción producida en un animal ante la presencia del hombre (Burrow y Dillon, 1997).

Si se asume que una respuesta al miedo por parte del animal apunta hacia el hombre, entonces se puede afirmar que los animales de temperamento nervioso (es decir, individuos con una mayor reacción adversa a los humanos) muestran una mayor respuesta al miedo en general. Las respuestas al miedo pueden surgir de las interacciones sociales, encuentros con nuevas especies y situaciones o estímulos repentinos que pueden ser visuales, auditivos o táctiles en la naturaleza (Curley Jr, 2004).

Estos animales de temperamento nervioso son susceptibles de presentar mayor estrés durante actividades rutinarias como la marcación, la vacunación, la vermifugación, la castración, el pesaje y el transporte; las cuales producen en el individuo experiencias de miedo, deshidratación, hambre y actividad física aumentada; que conllevan a producir fatiga y lesiones en los animales (Ferguson y Wagner, 2008).

Estos factores estresantes alteran la homeostasis en el animal, provocando una respuesta adaptativa que es activada en un intento por restaurar el balance a través de una serie de mecanismos fisiológicos que involucran el eje Hipotalámico-Hipofisiario-Adrenal (Moberg, 2000) y dependiendo de la magnitud del estímulo, duración e intensidad de los estresores y la susceptibilidad de los animales hacia ellos, se puede presentar inhibición del sistema inmunológico que predispone posteriormente a que se afecte la fisiología durante el crecimiento y la reproducción en los bovinos (Burdick et al., 2011; Fell et al., 1999).

Esta alteración afecta negativamente los rendimientos de producción en el hato ganadero, entre los cuales se destacan: las bajas ganancias diarias de peso (GDP), que conllevan a aumentar el tiempo de ceba de los animales, ocasionando pérdidas económicas para el productor.

Varios trabajos han mostrado que los bovinos de temperamento nervioso también presentan bajos resultados en la evaluación de la calidad de la canal y de la carne, ocasionados por aumento en el tamaño y frecuencia de hematomas, valores de $\mathrm{pH}$ en la canal por encima de 5.8, cortes de carne oscura y carne con menor terneza en comparación con aquellos animales de temperamento calmado, ocasionando de esta forma pérdidas en el rendimiento de la canal y disminu- 
ción en la calidad organoléptica y sensorial de la carne (Apple et al., 2005; Burrow and Dillon, 1997; Cafe et al., 2011; Fell et al., 1999; Fordyce et al., 1988; King et al., 2006).

Durante la evaluación del temperamento, los animales excitables (nerviosos) obtienen puntajes mayores a partir de evaluaciones subjetivas (evaluación de temperamento en corral y brete) y objetivas (evaluación de velocidad de salida del brete) de temperamento, con relación al de aquellos animales de temperamento calmado (Burrow and Dillon, 1997; Fordyce et al., 1988; Petherick et al., 2002). Asimismo, algunas evaluaciones de VSB se han correlacionado negativamente con el peso de la canal bovina (Cafe et al., 2011).

Teniendo en cuenta lo expuesto anteriormente, el propósito de este artículo fue revisar el conocimiento actual del impacto que tiene el temperamento sobre el crecimiento (relacionado con las ganancias de peso - GDP), la calidad de la canal y de la carne, con el fin de reducir las pérdidas económicas en la producción de ganado bovino de carne.

\section{El estrés y factores que inciden en éste}

Los organismos vivos se esfuerzan por mantener la homeostasis a nivel celular; sin embargo, cuando las condiciones celulares son alteradas por un factor externo, el individuo intenta restaurar su estado homeostático a través de una serie de mecanismos fisiológicos (Falkenberg, 2006).

Un factor que puede provocar esa respuesta celular es el estrés. El termino estrés se refiere a la suma de todas las reacciones biológicas, físicas, emocionales o mentales que alteran la homeostasis del individuo (Pacák and Palkovits, 2001). Como consecuencia de este estado alterado, se produce una respuesta que es activada con el fin de ayudar al cuerpo a afrontar la amenaza, para mantener el equilibrio.

Estas reacciones en el individuo se realizan a través de circuitos centrales, que están genéticamente programados y modulados por factores medioambientales, principalmente a través del eje Hipotalámico-Pituitario-Adrenal (HPA) y el Sistema Nervioso Simpático (SNS) (Elenkov et al., 2000; Pacák and Palkovits, 2001).

\section{Eje hipotalámico pituitario-adrenal (HPA)}

La activación del eje HPA inducido por el factor de estrés, produce una cascada de eventos mediados por sustancias endocrinas que permiten hacer frente al estímulo estresante. Como respuesta a los estímulos estresantes, los centros superiores del cerebro estimulan las neuronas en el Núcleo Paraventricular (NPV) del hipotálamo, para que produzcan la liberación de la hormona liberadora de corticotropina (HLC) y vasopresina (VP) (Carrasco and Van de Kar, 2003).

Cuando la HLC y la VP aumentan en la circulación portal hipotalámica-hipofisiaria, son expuestas a las hormonas corticotropas de la glándula pituitaria anterior que activan el eje adrenal. Después de la estimulación realizada por la HLC y la VP, se produce síntesis y secreción de la hormona adrenocorticotropa (ACTH) y su liberación en la circulación periférica permite la activación de la corteza suprarrenal, para producir glucocorticoides (GCs) y catecolaminas (Curley Jr, 2004). En el caso del ganado bovino, el primer glucocorticoide (GC) asociado con respuesta al estrés es el cortisol.

Como los GCs aparecen al final de la respuesta del eje HPA, las concentraciones de suero o plasma del GC se pueden utilizar como indicadores fisiológicos presentes durante la respuesta al estrés en un individuo.

\section{Sistema nervioso simpático (SNS)}

Ante la presencia de muchos estresores, el SNS es activado a través de neuronas noradrenérgicas en el cerebro y de neuronas simpáticas postganglionares que inervan los órganos periféricos (ejemplo: Corazón, vasculatura, riñones, intestino y tejido adiposo), secretando de esta forma norepinefrina dentro de la circulación, que aumenta la presión sanguínea y las frecuencias cardiaca y respiratoria (Burdick et al., 2011).

Un aumento de las concentraciones de norepinefrina en el cerebro, sirve como un sistema de alarma, el cual resulta en una disminución de las actividades neurovegetativas (ejemplo: comer y dormir) y en la activación del eje HPA. También se produce liberación de serotonina y acetilcolina las cuales activan el NPV para producir HLC (Black, 2002).

\section{Respuesta neuroendocrina}

El reconocimiento de una respuesta de estrés implica dos rutas principales. La primera es la activación del eje HPA inducido por el estrés, la cual resulta en una serie de eventos que permiten al individuo hacer frente al estímulo estresante. La segunda vía más importante de la mediación fisiológica de la respuesta al estrés es la activación del eje simpático-adrenal (Minton et al., 1992). Esto resulta en la liberación de los neurotransmisores adrenérgicos, la epinefrina y la norepinefrina, a partir de los nervios simpáticos y medulares de las glándulas suprarrenales. 
La primera onda que se produce casi inmediatamente en respuesta a un factor de estrés implica: 1) aumento de la secreción de catecolaminas (epinefrina y norepinefrina) del sistema nervioso simpático; 2) liberación hipotalámica de la hormona liberadora de corticotropina (HLC) en la circulación portal y segundos más tarde, aumento de la secreción de ACTH hipofisaria; 3) disminución de la liberación hipotalámica de la hormona liberadora de gonadotropina $(\mathrm{GnRH})$ y poco después, disminución de la secreción de gonadotropina pituitaria; 4) disminución de la hormona de crecimiento $(\mathrm{GH})$, y de la secreción pancreática de glucagón (Gerrard and Grant, 2002). Luego de unos minutos, una segunda onda más lenta implica la estimulación de la secreción de GCs y se suprime la secreción gonadal de esteroides (Falkenberg, 2006).

\section{Efectos hormonales}

Durante el estrés, el individuo responde a través de mecanismos hormonales que logran producir cambios fisiológicos importantes en el organismo. En unos pocos minutos estos cambios incluyen: 1) desviación de la energía a los músculos en forma de movilización de energía almacenada y disminución de la gluconeogénesis; 2) estimulación de la función inmune; 3 ) inhibición de la fisiología reproductiva y 4) disminución del apetito (Sapolsky et al., 2000).

Otros cambios fisiológicos incluyen: taquicardia, aumento de la frecuencia respiratoria, elevación de la temperatura corporal y la redistribución del volumen sanguíneo visceral hacia el músculo esquelético y el cerebro. En cuanto al comportamiento, son observados algunos cambios como: aumento de la alerta, agresión y escape (Ferguson and Warner, 2008).

Inicialmente los mediadores endocrinos involucrados en la respuesta al estrés, estimulan la concentración de glucosa en la sangre, en parte por la movilización de los depósitos existentes, y por una rápida resistencia a la insulina. Por consiguiente la energía se desvía desde los sitios de almacenamiento en el músculo por acción de las catecolaminas, glucagón y la GH (Black et al., 1982).

Por otro lado, el mecanismo simpático - adrenal de la respuesta autonómica, está mediado por las catecolaminas (epinefrina y norepinefrina) y los GCs (ej. el cortisol) de la corteza adrenal (Ferguson and Warner, 2008).

\section{- Catecolaminas}

En el metabolismo energético la secreción de catecolaminas produce cambios significativos en la lipólisis, gluco- genolisis y la gluconeogénesis en los músculos (Kuchel, 1991). Ellos moderan la demanda de glucosa por parte de algunos tejidos, mientras que simultáneamente estimulan la gluconeogénesis hepática, para aumentar la entrega de glucosa al músculo esquelético y al cerebro.

Se ha sugerido que un aumento en la secreción endógena de catecolaminas produce un aumento en la utilización de glucógeno intramuscular (Foury et al., 2005; Lowe et al., 2002) y además en este proceso podrían agotarse completamente las reservas de grasa del músculo (Lowe et al., 2004). Por otro lado, las catecolaminas funcionan para regular ciertas funciones corporales. Sin embargo, cuando el animal es sometido a algún factor estresante, las catecolaminas aumentan la frecuencia cardiaca, la presión sanguínea y las concentraciones de ácidos grasos libres (Shaw and Tume, 1992).

\section{- Glucocorticoides}

El efecto más notable de los GCs sobre el metabolismo es su capacidad para aumentar las concentraciones circulantes de glucosa. Los GCs son una clase de hormonas derivadas del colesterol que estimulan la gluconeogénesis, la proteólisis de los tejidos musculares, y la lipólisis de los tejidos adiposos (Sherwood, 2008). Estos cambios metabólicos aseguran que el cuerpo del individuo tenga la energía suficiente para hacer frente al estrés.

\section{Importancia del temperamento en la producción de carne}

Con la domesticación de los animales y con el pasó de los años e investigaciones realizadas, se han observado respuestas de comportamiento individuales frente a la presencia del hombre, que difieren mucho de una especie a otra y también dentro de las manadas. Scott y Fredericson (1951) se refirieron a "docilidad" y "salvaje", como la forma en la que reaccionan los animales al hombre y además, definieron el término "mansedumbre" como la ausencia de conducta conflictiva, y el término "salvaje" como la tendencia a escapar.

Esta caracterización ha permitido que el hombre pueda identificar a aquellos individuos que son más fáciles de trabajar en comparación con los que necesitan precauciones que deben tomarse al momento del manejo. El término "temperamento" también se ha utilizado para caracterizar las respuestas de comportamiento del animal ante la presencia del hombre (Burrow and Dillon, 1997). 
En los animales estas respuestas de comportamiento pueden variar desde manifestaciones de docilidad hasta nerviosismo (miedo), desde la falta de respuesta mostrada por inmovilidad para escapar, hasta la conducta agresiva o de ataque (Stricklin and Kautz-Scanavy, 1984).

Como se mencionó anteriormente, el término "temperamento" se define como la reacción producida en un animal ante la presencia del hombre, ya que se asume que una respuesta al miedo por parte del animal apunta hacia el hombre, entonces se puede afirmar que los animales de temperamento nervioso muestran una mayor respuesta al miedo que surgen de las interacciones sociales, encuentros con nuevas especies y situaciones o estímulos repentinos que pueden ser visuales, auditivos o táctiles en la naturaleza (Curley Jr, 2004).

\section{Evaluación del temperamento}

La selección de bovinos con temperamento más calmado, se traducirá en animales con menos estrés durante las actividades de manejo habituales en la explotación ganadera, permitiendo de esta forma reducir el riesgo para el personal encargado del manejo de los animales (Vann et al., 2012).

En la actualidad existen diferentes metodologías para evaluar el temperamento de los bovinos, las cuales se clasifican en evaluaciones subjetivas (evaluación en corral y en brete) y objetivas (evaluación de salida del brete). Estas mediciones se pueden unificar para generar un índice de temperamento de corral $(\mathrm{ITC}=\mathrm{EC}$ + VSB / 2) ó de brete (ITB= EB + VSB / 2), que permiten clasificar a los animales como calmados, intermedios y nerviosos (King et al., 2006).

La respuesta del estrés a estas pruebas dependerán del entorno en donde se realicen; es decir, que cada animal puede ver una situación en particular como estresante o no estresante, en comparación con otro individuo, por tal motivo es necesario realizar varias observaciones durante la evaluación del temperamento (Grandin, 1993).

\section{- Evaluación en el corral (EC)}

La EC permite que el animal se mueva libremente en el corral en presencia de un evaluador. Este método fue desarrollado por Hammond et al., (1996) y utiliza una escala de 1 a 5, donde el mayor puntaje corresponde a los animales nerviosos y el menor a los calmados. La escala propuesta es así: (1) el animal camina lentamente, no se asusta por la presencia del evaluador;
(2) corre hacia las cercas y se ubica en las esquinas ante la presencia del evaluador; (3) corre hacia la cerca, cabeza alta, y corre si el evaluador se acerca más, se detiene antes de golpear puertas y cercas; (4) corre, se ubicaba en la parte de atrás del grupo de animales, cabeza elevada, y muy atento a la presencia del evaluador, corre hacia puertas y cercas; (5) animal muy nervioso, corre hacia las cercas, y se mueve permanentemente ante cualquier estímulo.

Durante este trabajo Hammond et al., (1996), encontraron una relación entre las evaluaciones en corral y las concentraciones de cortisol. Ellos también notaron que el tiempo requerido para manejar a los bovinos en la manga y el brete, tuvo efectos significativos en la evaluación del temperamento de los animales.

\section{- Evaluación en el brete (EB)}

Este tipo de evaluación inhibe el movimiento natural del animal y mide la respuesta del animal ante la restricción. El sistema mide la cantidad de movimientos que el animal realiza y puede empezarse a realizar cerca del destete, ya que el comportamiento del animal puede ser alterado por experiencias anteriores, y además puede ser usado hasta finalizar la ceba, para observar los cambios de temperamento del animal. El animal debe ser evaluado sin restricción aplicada cuando se encuentre dentro del brete. El método incluye una escala de 1 a 5, donde; (1) calmado, no movimiento; (2) levemente agitado; (3) golpes ocasionales de la calceta; (4) movimiento continuo y vigoroso con golpes de la calceta; (5) movimiento violento y giros dentro de la calceta (Grandin, 1993).

King et al., (2006) utilizaron la EB y encontraron que fue menos efectiva en diferenciar el temperamento de los animales; mientras que otros autores tuvieron mejor respuesta al utilizar el método para categorizar al temperamento y encontraron fuertes relaciones entre esos grupos y resultados de calidad de la carne bovina (Voisinet et al., 1997; Wulf, 1997).

\section{- Velocidad de salida del brete (VSB)}

Burrow et al., (1988) describieron un método más objetivo para evaluar el temperamento y fue definido como la velocidad de salida del brete (VSB). Esta prueba consiste en medir el tiempo de salida del brete y se realiza mediante un sistema electrónico infrarrojo, que se conecta a una unidad de registro, una vez que el animal pasa por el sensor 1 (ubicado a 1 metro de la salida del brete), se enciende un cronómetro que se 
detiene cuando el animal pasa por el sensor 2 (ubicado a 1.83 metros del sensor 1 ).

Uno de los trabajos realizados (Curley Jr, 2004) determinaron que la VSB está correlacionada con la evaluación en corral y brete. Además, la VSB estuvo correlacionada con concentraciones de cortisol en la sangre. En otro estudio Schwartzkopf-Genswein et al., (2012) realizaron comparación de diferentes técnicas para evaluar el temperamento y encontraron que la VSB y la evaluación visual en el brete se correlacionan.

León-Llanos et al., (2012a), evaluaron el temperamento de bovinos Bos indicus y Bos indicus x Bos taurus mediante la EC, EB y VSB. Este trabajo demostró que el índice de temperamento del brete (EB + VSB / 2), logró categorizar a los animales como calmados, intermedios y nerviosos. Por otra parte la EC no produjo diferencias significativas para categorizar a los animales como calmados e intermedios; sin embargo, hubo diferencias significativas en la EC entre los animales calmados y nerviosos.

Hay que tener presente que algunos aspectos del temperamento como la agresión, no son objeto de la VSB. El objetivo principal de la medida busca evaluar la respuesta del animal al miedo y a la angustia de estar restringido y separado del grupo, ya que el miedo es un estresor muy fuerte y los resultados altamente variables durante el manejo son probablemente debidos a diferentes niveles de estrés sicológico en el animal (Burrow et al., 1988; Grandin, 1997; Vann et al., 2012).

\section{Efecto del temperamento sobre el crecimiento}

Hasta ahora se tiene entendido que el temperamento y el estrés han estado estrechamente asociados, y que la evaluación del temperamento del ganado se ha convertido en un método para seleccionar a los animales que podrían ser menos sensibles al estrés o que se adaptan mejor a las condiciones de manejo, permitiendo de esta forma mejorar la productividad en la explotación (Curley Jr, 2004).

Por ejemplo, se ha demostrado que el tiempo de alimentación y consumo de materia seca (MS) es reducido en animales que obtuvieron mayor VSB y que fueron clasificados como nerviosos (Cafe et al., 2011). Igualmente se han obtenido correlaciones negativas entre el puntaje en la EB y VSB, frente a las GDP en bovinos (Hoppe et al., 2010).

En síntesis aquellos animales clasificados como nerviosos presentan disminución en el crecimiento; es decir, que tienen GDP bajas frente a la de animales con temperamento calmado (Cafe et al., 2011; Fell et al., 1999;
Müller and von Keyserlingk, 2006). Por tal motivo los animales con temperamento calmado presentan mayores pesos vivos que los animales nerviosos (Fordyce et al., 1985).

En el Piedemonte del Meta, León-Llanos et al., (2012a) encontraron que los bovinos clasificados con temperamento intermedio, presentaron menor GDP durante el levante en comparación con los bovinos calmados, pero no encontraron diferencias durante la fase de ceba. Sin embargo, los bovinos nerviosos tuvieron mayor edad al sacrificio en comparación con animales de temperamento intermedio y menor peso al sacrificio y peso vivo después del ayuno previo al sacrificio en comparación con los animales calmados; asimismo, la pérdida de peso y porcentaje en ayuno, fueron mayores en bovinos intermedios en comparación con bovinos calmados.

Los resultados anteriores indicarían que existe una influencia positiva del temperamento en aquellos animales destetos calmados, sobre el crecimiento durante el levante. También los resultados muestran que los bovinos con temperamento nervioso presentaron mayor tiempo de ceba, menor peso al sacrificio y mayor pérdida de peso por el ayuno. Lo cual significa que es recomendable evaluar y seleccionar animales de temperamento calmado durante la etapa de levante, lo que permitirá facilitar el manejo y mejorar el crecimiento de los bovinos hasta la etapa de ceba (LeónLlanos et al., 2012a).

Una de las hipótesis que se tiene con respecto al crecimiento lento del ganado de temperamento nervioso, es que ellos realizan un mayor gasto de energía, el cual está asociado con un comportamiento más vigilante, causando un menor consumo o tasa de conversión de alimento (Burrow and Dillon, 1997; Petherick et al., 2002).

En bovinos Brahman de Nuevo Gales del Sur (Australia), el aumento de los valores de VSB (metros/segundo) durante seis meses de pastoreo antes de la etapa de ceba, se asociaron con la reducción en el consumo de alimento de 370 gramos de MS/día, y la disminución de 4.7 minutos/día en la cantidad de tiempo dedicado para alimentarse (Cafe et al., 2011).

\section{Efecto del temperamento sobre la calidad de la canal y de la carne}

Por lo general se ha visto que los animales de temperamento nervioso pueden afectar las características de calidad de la canal por aumento en la frecuencia y tamaño de hematomas y valores de $\mathrm{pH}$ por encima de 
5.8 (después de 24 horas), ocasionando una disminución en la calidad organoléptica y sensorial de la carne (Burrow and Dillon, 1997; Cafe et al., 2011; Fell et al., 1999; Fordyce et al., 1988; King et al., 2006). Además aquellos animales que obtienen evaluaciones de VSB mayores han presentado canales con peso bajo (Cafe et al., 2011).

En frigoríficos del Meta y Casanare, León-Llanos (2009) encontró que las lesiones principales en la canal fría correspondieron a hematomas (75\%), cortes oscuros $(13 \%)$, equimosis $(9 \%)$, fracturas $(2 \%)$ y abscesos $(1 \%)$ y que además el $76.8 \%$ de los animales poseían cuernos al momento del sacrificio, principalmente en bovinos Bos indicus. Indicando posiblemente que la presencia de cuernos en ganado bovino Bos indicus, predispone a que los animales se vuelvan temperamentales y manifiesten estrés fácilmente durante el manejo y el transporte, ocasionando mayor ocurrencia de golpes, heridas y lesiones en la canal.

Uno de los defectos que más afectan la calidad de la canal son los cortes oscuros, que se caracterizan por elevado $\mathrm{pH}$ y alta capacidad de retención de agua (Ribeiro et al.), por consiguiente la carne de estos animales se volverá seca, firme, pegajosa y con un color entre rojo oscuro y casi negro (DFD). La responsable de esta deplorable condición en las canales bovinas es la disminución de la reserva muscular de glucógeno antes del sacrificio, la cual es observada en animales de temperamento nervioso o que han sido sometidos a largas horas de transporte hasta el frigorífico (Apple et al., 2005).

En el Piedemonte del Meta se encontró que los bovinos de temperamento nervioso presentaron mayor valor de $\mathrm{pH}$ en la canal a las 24 horas de refrigeración en comparación con los bovinos intermedios y calmados. Este valor pudo haber influenciado los resultados de baja terneza, humedad, porcentaje de agua unida, luminosidad y alta CRA en comparación con los de bovinos calmados (León-Llanos et al., 2012a; León-Llanos et al., 2012b).

Purchas y Aungsupakorn (1993), afirmaron que la carne más dura corresponde a canales que presentan el $\mathrm{pH}$ entre 5.8 y 6.2 ; mientras que cuando el $\mathrm{pH}$ de la canal a las 24 horas se encuentra alrededor de 5.6, algunas enzimas como las catepsinas encuentran el punto óptimo para realizar actividad proteolítica, ocasionado mejor terneza en la carne (de Oliveira Roça, 2009).

Sin embargo, no se puede decir lo mismo para la correlación genética $\left(r_{g}=0.3-0.4\right)$ entre el temperamento y la terneza de la carne bovina, indicando que la selección del temperamento basado en la evaluación de la VSB, podría ser una herramienta importante para realizar mejoramiento genético en bovinos destinados a producir carne con una buena terneza (Kadel et al., 2006).

El temperamento es un rasgo de heredabilidad mediana y algunas asociaciones de razas Angus y Limousin en Estados Unidos están utilizando diferencias esperadas de progenie de docilidad (DEP) que reflejarán la probabilidad de que la descendencia herede genes asociados a temperamento más tranquilo (Beckman, 2008).

León-Llanos et al., (2012b), encontraron que la carne de bovinos de temperamento nervioso, presentaron menor luminosidad en comparación con animales de temperamento intermedio y calmado. La intensidad del color de la carne está influenciada por el $\mathrm{pH}$ y la morfología de la estructura muscular. Como se ha mencionado anteriormente, el agotamiento de glucógeno producirá un $\mathrm{pH}$ final alto en la canal de los animales de temperamento nervioso y la carne tenderá a tener una apariencia muy oscura por agotamiento de los nutrientes; es decir, que los animales no tendrán suficiente glucosa o glucógeno presente en el músculo para disminuir el $\mathrm{pH}$ por la glucólisis en ácido láctico. De este modo, las fibras musculares de corte oscuro estarán hinchadas y pegadas unas de otras formando barreras que evitan que haya absorción de luz y difusión de oxígeno para que se combine con la mioglobina y formen la oximioglobina (Seideman et al., 1984).

\section{Conclusión}

La recopilación de diferentes trabajos expuestos en el presente artículo, indican que el ganado bovino que exhibe temperamento nervioso tiende a disminuir las GDP, afectando el normal desarrollo durante la etapa de crecimiento. Además, muestran mayor reactividad al estrés cuando se realizan evaluaciones de temperamento (EC, EB y VSB) y han sido correlacionadas con hormonas como cortisol, epinefrina y norepinefrina, lo cual indica que el uso de estas herramientas pueden ser claves al momento de seleccionar animales para el levante y la ceba, permitiendo mejorar los índices de productividad en las explotaciones ganaderas.

No es recomendable llevar animales temperamentales hasta la etapa de ceba, ya que son más susceptibles de presentar canales con mayor número y tipo de lesiones (hematomas, fracturas, abscesos y cortes oscuros) y con valores elevados de $\mathrm{pH}$ a las 24 horas, afectando la terneza, humedad, porcentaje de agua unida, lumi- 
nosidad y CRA de la carne en comparación con la de bovinos calmados.

Para el productor, la medición del temperamento puede ser una herramienta útil en la selección de hembras y machos de reemplazo, lo cual ayudaría a evitar la presencia de novillas y toretes más nerviosos que podrían transmitir ese aspecto negativo a generaciones futuras.

\section{Bibliografía}

Apple JK, Kegley EB, Galloway DL, Wistuba TJ, Rakes LK,. Duration of restraint and isolation stress as a model to study the darkcutting condition in cattle. J Anim Sci. 2005;83:1202-1214.

Beckman DW. 2008. Docility EPD: A Tool for Temperament.

Black PH. 2002. Stress and the inflammatory response: a review of neurogenic inflammation. Brain, behavior, and immunity.

Black PR, Brooks DC, Bessey PQ, Wolfe RR, Wilmore DW. Mechanisms of insulin resistance following injury. Ann Surg. 1982;196(4):420-435.

Burdick NC, Randel RD, Carroll JA, Welsh TH. 2011. Interactions between Temperament, Stress, and Immune Function in Cattle. International Journal of Zoology 2011.

Burrow H, Seifert G, Corbet N. A new technique for measuring temperament in cattle. In, Australia. Proc. Aust. Soc. Anim. Prod. 1988;17:154-157.

Burrow HM, Dillon RD. Relationships between temperament and growth in a feedlot and commercial carcass traits of Bos indicus crossbreds. Aust J Exp Agric. 1997;37:407-411.

Cafe LM, Robinson DL, Ferguson DM, Mclntyre BL, Geesink $\mathrm{GH}$, Greenwood PL. Cattle temperament: Persistence of assessments and associations with productivity, efficiency, carcass and meat quality traits. J Anim Sci. 2011;89:1452-1465.

Carrasco GA, Van de Kar LD. Neuroendocrine pharmacology of stress. Eur J Pharmacol. 2003;463(1-3):235-272.

Crouse JD, Cundiff LV, Koch RM, Koohmaraie M, Seideman SC. Comparisons of Bos Indicus and Bos Taurus Inheritance for Carcass Beef Characteristics and Meat Palatability. J Anim Sci. 1989;67:2661-2668.

Curley Jr O. 2004. Influence of temperament on bovine hypothalamic-pituitary-adrenal function. Master of Science. Texas A\&M University, de Oliveira Roça, R. 2009. Modificações post-mortem (F.C.A. - UNESP - Campus de Botucatu).

Elenkov IJ, Wilder RL, Chrousos GP, Vizi ES. The Sympathetic NerveAn Integrative Interface between Two Supersystems: The Brain and the Immune System. Pharmacol Rev. 2000;52(4):595-638.

Falkenberg SM. 2006. Stress response effects on growth, carcass characteristics, and tenderness in Bonsmara-influenced steers. Texas A\&M University.

Fell LR, Colditz IG, Walker KH, Watson DL. Associations between temperament, performance and immune function in cattle en- tering a commercial feedlot. Aust J Exp Agric. 1999;39(7):795802.

Ferguson DM, Warner RD. Have we underestimated the impact of pre-slaughter stress on meat quality in ruminants? Meat Science. $2008 ; 80: 12-19$.

Fordyce G, Goddard ME, Tyler R, Williams G, Toleman M. Temperament and bruising of Bos indicus cattle. Australian Journal of Experimental Agriculture and Animal Husbandry. 1985;25:283-288.

Fordyce G, Wythes J, Shorthose W, Underwood D, Shepherd R. Cattle temperaments in extensive beef herds in northern Queensland. 2. Effect of temperament on carcass and meat quality. Aust J Exp Agric. 1988;28(6):689-693.

Foury A, Devillers N, Sanchez MP, Griffon H, Le Roy P, Mormède P. Stress hormones, carcass composition and meat quality in Large White x Duroc Pigs. Meat Science. 2005;69(4):703-707.

Gerrard DE, Grant AL. 2002. Principles of animal growth and development. Kendall Hunt.

Grandin T. Behavioral agitation during handling of cattle is persistent over time. Appl Anim Behav Sci. 1993;36(1):1-9.

Grandin T. Assessment of stress during handling and transport. J Anim Sci. 1997;75:249-257.

Hammond AC, Olson TA, Chase CC, Bowers EJ, Randel RD, Murphy $\mathrm{CN}$, Vogt DW, Tewolde A. Heat tolerance in two tropically adapted Bos taurus breeds, Senepol and Romosinuano, compared with Brahman, Angus, and Hereford cattle in Florida. J Anim Sci. 1996;74(2):295-303.

Hoppe S, Brandt HR, König S, Erhardt G, Gauly M. Temperament traits of beef calves measured under field conditions and their relationships to performance. J Anim Sci. 2010;88(6):19821989. doi: 10.2527/jas.2008-1557.

Kadel MJ, Johnston DJ, Burrow HM, Graser H, Ferguson DM. Genetics of flight time and other measures of temperament and their value as selection criteria for improving meat quality traits in tropically adapted breeds of beef cattle. Aust J Agric Res. 2006;57(9):1029-1035.

King DA, Schuehle Pfeiffer CE, Randel RD, Welsh JTH, Oliphint RA, Baird BE, et al. Influence of animal temperament and stress responsiveness on the carcass quality and beef tenderness of feedlot cattle. Meat Science 2006;74(3):546-556.

Kuchel O. Stress and catecholamines. Methods Achiev Exp Pathol 1991;14:80-103.

León-Llanos LM. 2009. Evaluación de la calidad de la canal y el ganado bovino en dos frigoríficos de la región de la Orinoquia. Universidad de los Llanos, Villavicencio, Meta.

León-Llanos LM, Ballesteros-Chavarro HH, Flórez-Díaz H. 2012a. Efecto del temperamento sobre el crecimiento y las características de la canal de bovinos Cebú y sus cruces en la Orinoquia colombiana. In XXIII Congreso Panamericano de Ciencias Veterinarias - Panvet 2012 (Cartagena de Indias), p. 1.

León-Llanos LM, Ballesteros-Chavarro HH, Flórez-Díaz H. 2012b. Efecto del temperamento y el transporte previo al sacrificio sobre las características instrumentales y sensoriales de la carne 
de bovinos Cebú y sus cruces en la Orinoquia colombiana. In XXIII Congreso Panamericano de Ciencias Veterinarias - Panvet 2012 (Cartagena de Indias), p. 1.

Lowe TE, Devine CE, Wells RW, Lynch LL. The relationship between postmortem urinary catecholamines, meat ultimate $\mathrm{pH}$, and shear force in bulls and cows. Meat Science. 2004;67(2):251260.

Lowe TE, Peachey BM, Devine CE. The effect of nutritional supplements on growth rate, stress responsiveness, muscle glycogen and meat tenderness in pastoral lambs. Meat Science. 2002;62(4):391-397.

Minton JE, Coppinger TR, Reddy PG, Davis WC, Blecha F. Repeated restraint and isolation stress alters adrenal and lymphocyte functions and some leukocyte differentiation antigens in lambs. J Anim Sci. 1992;70(4):1126-1132.

Moberg G. 2000. Biological response to stress: implications for animal welfare. In The biology of animal stress - Basic principles and implications for animal welfare, Mench, G.P.M.J.A., ed. (Oxon, UK, CABI Publishing), pp. 1-21.

Müller R, von Keyserlingk MAG. Consistency of flight speed and its correlation to productivity and to personality in Bos taurus beef cattle. Appl Anim Behav Sci. 2006;99(3-4):193-204.

Pacák K, Palkovits M. Stressor Specificity of Central Neuroendocrine Responses: Implications for Stress-Related Disorders. Endocr Rev. 2001;22(4):502-548.

Petherick JC, Holroyd RG, Doogan VJ, Venus BK. Productivity, carcass and meat quality of lot-fed Bos indicus cross steers grouped according to temperament. Aust J Exp Agric. 2002;42(4):389398.

Purchas RW, Aungsupakorn R. Further investigations into the relationship between ultimate $\mathrm{pH}$ and tenderness for beef samples from bulls and steers. Meat Science 1993;34(2):163-178.

Ribeiro JdS, Gonçalves TdM, Ladeira MM, Tullio RR, Campos FR, Bergmann JAG, et al., Reactivity, performance, color and tenderness of meat from Zebu cattle finished in feedlot. Rev Bras Zootec. 2012;41(4):1009-1015.
Santana DA. 2009. Competir e innovar la ruta de la industria bovina: agenda prospectiva de investigación y desarrollo tecnológico para la cadena cárnica bovina en Colombia.

Sapolsky RM, Romero LM, Munck AU. How Do Glucocorticoids Influence Stress Responses? Integrating Permissive, Suppressive, Stimulatory, and Preparative Actions. Endocr Rev. 2000;21(1):55-89.

Scott J, Fredericson E. The causes of fighting in mice and rats. Physiol Zool. 1951;14(4):273-309.

Schwartzkopf-Genswein KS, Shah MA, Church JS, Haley DB, Janzen K, Truong G, Atkins RP, Crowe TG. A comparison of commonly used and novel electronic techniques for evaluating cattle temperament. Can J Anim Sci. 2012;92(1):21-31.

Seideman SC, Cross HR, Smith GC, Durland PR. Factors associated with fresh meat color: A Review. Journal of Food Quality. 1984;6:211-237.

Shaw FD, Tume RK. The assessment of pre-slaughter and slaughter treatments of livestock by measurement of plasma constituents-A review of recent work. Meat Science 1992;32:311329.

Sherwood L. 2008. Human Physiology: From Cells to Systems. Brooks/Cole, Cengage Learning.

Stricklin WR, Kautz-Scanavy CC. The role of behavior in cattle production: A review of research. Applied Animal Ethology. 1984;11:359-390.

Vann R, Randel R, Welsh Jr T. 2012. Tools for Assessing Temperament in Beef Cattle. Division of Agriculture, Forestry and Veterinary Medicine Mississippi State University October 2012. Animal and Dairy Sciences. P.p. 91-95

Voisinet BD, Grandin T, O'Connor SF, Tatum JD, Deesing MJ. Bos indicus-cross feedlot cattle with excitable temperaments have tougher meat and a higher incidence of borderline dark cutters. Meat Science 1997;46:367-377.

Wulf DM, O'Connor SF, Tatum JD, Smith GC. Using objective measures of muscle color to predict beef longissimus tenderness. Journal of Animal Science. 1997;75:684-692. 